\title{
Library Function Tracking with XALT
}

\author{
Reuben D. Budiardja \\ Kapil Agrawal \\ University of Tennessee \\ Knoxville \\ reubendb@utk.edu \\ kagrawa1@vols.utk.edu
}

\author{
Mark Fahey \\ Argonne National Laboratory \\ mfahey@anl.gov
}

\author{
Robert McLay \\ Doug James \\ Texas Advanced Computing \\ Center \\ mclay@tacc.utexas.edu \\ djames@tacc.utexas.edu
}

\begin{abstract}
$\mathrm{XALT}$ is a tracking tool that collects accurate, detailed, and continuous job-level and link-time data. XALT stores that data in a database and ensures that all the data collection is transparent to the users. XALT tracks libraries and object files linked by the application. A recent feature improvement in XALT enable it to also track external subroutines and functions called by an application. This paper describes the function-tracking implementation in XALT and showcases the kind of data and analysis that becomes available from this new feature. A recently developed web-based interface to XALT database is also described, allowing the staffs of a supercomputing center to more easily understand software usage on their compute resources.
\end{abstract}

\section{Keywords}

XALT user environment, library tracking, job analytics, software survey

\section{CCS Concepts}

-General and reference $\rightarrow$ Surveys and overviews; -Human-centered computing $\rightarrow$ Open source software; Empirical studies in collaborative and social computing; •Social and professional topics $\rightarrow$ User characteristics; •Software and its engineering $\rightarrow$ Software maintenance tools; Software organization and properties;

\section{INTRODUCTION}

XALT is a tool that collects accurate, detailed, and continuous job-level and link-time data and stores that data in a database; all the data collection is transparent to the users [1]. XALT tracks libraries and object files linked by the application. XALT was developed to provide answers to common questions faced by most centers of High Performance Computing (HPC), such as: How many users and projects

Publication rights licensed to ACM. ACM acknowledges that this contribution was authored or co-authored by an employee, contractor or affiliate of the United States government. As such, the Government retains a nonexclusive, royalty-free right to publish or reproduce this article, or to allow others to do so, for Government purposes only.

XSEDE16, July 17 - 21, 2016, Miami, FL, USA

(C) 2016 Copyright held by the owner/author(s). Publication rights licensed to ACM. ACM ISBN 978-1-4503-4755-6/16/07 . .\$15.00 DOI: http://dx.doi.org/10.1145/2949550.2949558 use a particular library or executable? How many users use the different versions of available compilers? Which center provided packages are used often and which ones are never used? Which users or applications still use old / obsolete versions of certain library, compiler, or executable? Are there any widely used user-installed packages that should instead be provided by the center?

Information collected by XALT can answer these types of software-related questions and more, giving insights to users' behavior and activities on the compute resource. Having this information means that the staffs of a computing center are able to provide better, more focused support, increasing productivity of the users and the staff themselves.

A recent feature improvement in XALT enables it to track external functions and subroutines needed by an application. The function-tracking capability allows XALT to have moreaccurate, finer-grain details about what an application uses. With its job-tracking capability, XALT generates a complete picture of compilers, libraries, subroutines, and even the environments needed by users to run their jobs successfully. The new function-tracking capability extends XALT capability and expands its benefits to the stakeholders of supercomputing facilities: the users, the staffs, the software developers, the vendors, and the funding agency.

In this paper we describe our implementation of XALT function-tracking feature. We begin with a general overview of how XALT works in $\S 2$. In $\S 3$ we describe the functiontracking feature and show some use cases for the data collected by this feature in particular. A recently developed web interface to access XALT data is described in $\S 4$. This is followed by concluding remarks in $\S 5$.

\section{XALT OVERVIEW}

The goal of XALT is to do an automatic, continuous, census of libraries and applications on supercomputers. It achieves this goal by collecting data at both link time and job-level for subsequent analysis.

For the link-time data, our approach is based on a wrapper that intercepts the linker (ld) at link time. Wrapping the linker is a clean and efficient way to intercept information automatically and transparently. During linking, XALT's linker collects the list of object files needed by the executable by parsing the user's link line and command line. The linker wrapper also climbs the process tree to find the process that calls the linker. In most cases, this is the compiler. This therefore provides some information of compiler usage and is recorded in the XALT database. While XALT's linker parses and stores data via one of XALT transmission meth- 
ods, the actual linker (e.g. GNU ld) is called with the same command arguments from the user to build the actual executable. During this process, an UUID $^{1}$ is generated and inserted as part of the ELF section header of the executable. This allows the executable to be identified during the job launch time, providing job-level data.

To collect job-level data, XALT provides wrappers for several common job launchers such as ibrun, mpirun, and aprun. If a wrapper for a particular job launcher is readily available, it is relatively simple to write one. When an executable is launched, the XALT job-launcher wrapper parses the command line arguments to get information such as the number of MPI tasks and the number of threads, records the runtime environment variables, and extracts the previously inserted XALT ELF header if exists. The wrapper stores this information via one of XALT's transmission methods and calls the actual job launcher to run the executable.

XALT provide three transmission methods to store information collected during link time and job launching. The file method stores XALT data in a hidden directory under the user's home directory /.xalt.d/ as $\mathrm{JSON}^{2}$ files. The syslog transfers the data via the common message logging facility on UNIX and Linux operating system. Both of these transmission methods require further post-processing to store the information into the XALT database. The directdb method allows the information to be stored directly into the database.

The development of XALT emphasizes several guiding principles:

- XALT must avoid impacting the user experience, therefore failure mode must be benign.

- XALT must be a lightweight solution with essentially no overhead at compilation, linking, and runtime.

- XALT must be extensible to support more than one linker and/or job launcher.

- XALT must work seamlessly on any cluster, workstation, or high-end supercomputer.

For a more complete description on how XALT works, its database design, and examples of data analysis and reporting, the reader is referred to [2].

\section{FUNCTION TRACKING}

Beyond understanding what software and libraries are used by applications, it is often desirable to also know what external functions or subroutines are called by an application. A recent version of XALT (version $>=0.7$ ) implements this so-called "function tracking" feature. In contrast, earlier versions only knows that an application uses, for example, the BLAS numerical library, but not which BLAS subroutines. The latest version of XALT (as of this writing) also detects the BLAS subroutines (e.g. dgemm, sgemv, etc.) that are actually called by the application. This capability becomes increasingly useful vendor-supplied libraries such as the Intel MKL, the Cray LibSci, or ACML combine multiple canonical numerical libraries (BLAS, LAPACK, Fourier

\footnotetext{
${ }^{1}$ Universally unique identifier, a 128-bit value used to uniquely identify information (see e.g.: https://tools.ietf.org/html/rfc4122.html)

${ }^{2}$ JSON is an open standard date interchange format, see http://json.org/
}

Transforms, etc) into a single package. The function tracking capability of XALT therefore allows a finer grain tracking of software usage on HPC systems. In this section we detail the implementation of this feature.

\subsection{Implementation}

For the function tracking feature, XALT needs to know the list of libraries (i.e. object files) whose functions we are interested in tracking. XALT uses the ReverseMap file (often shortened as rmap) to get this list. ReverseMap is a JSON-formatted file that maps the location of libraries and object files (i.e. directory paths) to the modulefile that provides them ${ }^{3}$. The ReverseMap file allows XALT to store easily-recognizable module name (i.e. software packages) associated with an application (either linked to or loaded during runtime). XALT uses Lmod utility called spider to build the ReverseMap as part as its installation step.

The ReverseMap file therefore already contains a list of directories in which libraries or object files reside (since they are provided by the modulefiles). A utility in XALT simply traverses these directories to build a unique list of object and archive files (i.e. .o, .a, and .so files) containing the functions / subroutines we should be interested in tracking. The list is stored back into an updated ReverseMap file. This step can be done during the XALT installation and repeated any time update the ReverseMap file (e.g. after a new software is installed or upgraded). To have the function tracking feature to work in XALT, a ReverseMap file is a requirement whereas it is optional in earlier XALT versions. If a ReverseMap file does not exist however, XALT does not produce any error; It simply will not track functions. This behavior is intentional and part of the design philosophy of XALT to avoid impacting the end-user experience as much as possible.

The real work of the function tracking occurs during the linking phase of the executable (i.e. application). Behind the scene XALT does a dual-linking process: the real linking as requested by the user (commonly via compiler or make) by the linker (e.g. GNU 1d), and an artificially failed linking created by XALT. The latter is done by omitting any object files that are in the ReverseMap file from the link line (i.e. the object files whose functions we want to track). This artificially failed linking would produce errors for unresolved symbols. These unresolved symbols are in fact the functions / subroutines called by the application that we want to track. Another utility in XALT parses the error messages and builds the list of tracked functions.

To avoid slowing down the end-user's linking process, the dual-linking is done asynchronously by forking a subshell to do the function-tracking failed linking and to parse of the unresolved symbols. This separate process also collects and transmits the data according to the chosen transmission method (see $\S 2$ ). Therefore the actual linking requested by the user can return as soon as it is done while any additional overhead of function tracking is hidden from the user. Additionally, the user is also shielded from experiencing any

\footnotetext{
${ }^{3}$ Environment Modules is a mechanism to manage user's environment dynamically and is commonly used in HPC to provide software or library packages. The definition of the user's environment for a certain software package is expressed in a modulefile. Common implementation of Environment Module includes the original project written in TCL [3] and TACC's Lmod [4]
} 
latency associated with the storing of XALT data.

\subsection{Use Cases and Results}

What information do we get from XALT function-tracking feature? What kind of insight can be gleaned from such information? What sort of use cases might this information be useful for? In this subsection we do an early explorations of those questions. Results and data here are obtained from XALT running on the Darter supercomputer and the Beacon cluster at the National Institute for Computational Sciences (NICS). To better illustrate the examples we show explicit SQL query required to gather the data. Some results have been obfuscated to protect user privacy.

The most obvious, if less interesting, case is to find the most called functions / subroutines. Listing 1 shows the SQL query for the XALT database needed to obtain the most called functions. Topping the list are subroutines/symbols that are part of language standards and therefore are called by most programs. As we go down further down the list we also see common MPI subroutines.

Listing 1: SQL query and its results for most called functions

SELECT lower(trim(function_name)) as FunctionName, count $(*)$ as count

FROM xalt_link xl, join_link_function lf, xalt_function $x f$

WHERE build_syshost $=$ [syshost]

AND $x l . l i n k_{-}$id $=$lf. link $k_{-}$id

AND lf.func_id $=x f$ func_id

GROUP BY FunctionName

ORDER BY count DESC

\begin{tabular}{|c|c|}
\hline FunctionName & $\operatorname{cnt}$ \\
\hline${ }_{--} \mathrm{gxx}_{-}$personality_v0 & 18021 \\
\hline std $::$ ios_base $::$ init $::$ init () & 17131 \\
\hline std $::$ ios_base $::$ init $::^{\sim}$ init () & 17131 \\
\hline operator & 16359 \\
\hline vtable & 14680 \\
\hline${ }_{--} \mathrm{cxa}_{-} \mathrm{pure} \mathrm{e}_{-} \mathrm{virtual}$ & 12994 \\
\hline$\cdots$ & \\
\hline mpi_finalize - & $\begin{array}{l}4714 \\
4676\end{array}$ \\
\hline $\begin{array}{l}\text { mpi_init_- } \\
\text { mpi_comm_rank }\end{array}$ & $\begin{array}{l}4676 \\
4590\end{array}$ \\
\hline $\begin{array}{l}\text { mpl_comm_rank- } \\
\text { mpi_comm_size }\end{array}$ & 4529 \\
\hline for_stop_core & 4517 \\
\hline for_write_seq_fmt_xmit & 4494 \\
\hline _intel_fast_memset & 4458 \\
\hline for $\_$close & 4398 \\
\hline _gfortran_st_read & 4273 \\
\hline _gfortran_st_read_done & 4273 \\
\hline _gfortran_transfer_real_write & 4270 \\
\hline mpi_barrier & 4179 \\
\hline _gfortran_st_open & 3999 \\
\hline - gfortran_transfer_real & 3994 \\
\hline for_read_seq_lis & 3975 \\
\hline for_read_seq_fmt & 3951 \\
\hline mpi_reduce_- & 3934 \\
\hline mpi_comm_rank & 3929 \\
\hline
\end{tabular}

A more interesting case is to find out what functions used from a package. For example, can we find out what subroutines are often used from library such as $\mathrm{FFTW}^{4}$ ? This question is relatively easy to answer due to the naming con-

\footnotetext{
${ }^{4}$ http://www.fftw.org
}

vention of FFTW's subroutines. They always have fftw_ prefix. Listing 2 shows the required SQL query and its results on Darter. From the results we can see that parallel FFTW routine with MPI and threads are often called by applications.

Listing 2: SQL query and its results for most used FFTW subroutines

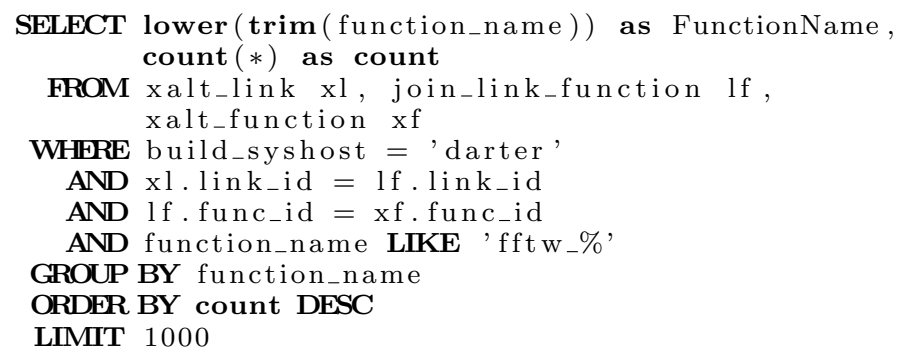

\begin{tabular}{|l|r|}
\hline FunctionName & count \\
\hline fftw_execute_dft & 425 \\
fftw_plan_dft_3d & 351 \\
fftw_malloc & 302 \\
fftw_execute & 295 \\
fftw_mpi_init & 191 \\
fftw_mpi_local_size_many & 187 \\
fftw_mpi_plan_dft & 187 \\
fftw_plan_with_nthreads & 146 \\
fftw_init_threads & 146 \\
fftwf_plan_many_dft & 119 \\
fftwf_destroy_plan & 119 \\
fftwf_execute_dft & 119 \\
fftwf_plan_many_dft_r2c & 115 \\
\hline
\end{tabular}

A similar but harder question to answer is to look for popular subroutines provided by software such as the Cray Scientific Library (also known as the Cray LibSci). The Cray Scientific Libraries provides subroutines from many other libraries such as BLAS, LAPACK, BLACS, ScaLAPACK, etc optimized for the Cray systems. To get at this question, we need to first obtained all the linking events that used the Cray LibSci, then obtain the distinct subroutines called by the executables in those linking events. On listing 3, the former is accomplished by the nested SQL SELECT statement. The latter is accomplished by the outer level SQL SELECT statement.

We need to do these two steps because there is no direct link in XALT database that connect libraries (object files) to their subroutines. The drawback of the above query is that we also get all subroutines belonging to all of the other libraries used in the linking events. Therefore, some heuristics are needed to filter out only the subroutines that belong to the Cray LibSci library. This can be done, for example, by getting a comprehensive list of the symbols provided by Cray LibSci and find the intersection of that list with the results of our query. For this particular illustration however we shall rely on the authors familiarity with this particular library to point out which subroutines in listing 3 belong to tge Cray LibSci, as indicated by their annotation in parentheses. From this listing it is clear to see that the BLAS and LAPACK routines for double-precision variables are most often called. 
Listing 3: SQL query and its results for most used Cray Libsci subroutines. Some subroutines have been removed (indicated by "...") from the list to save space.

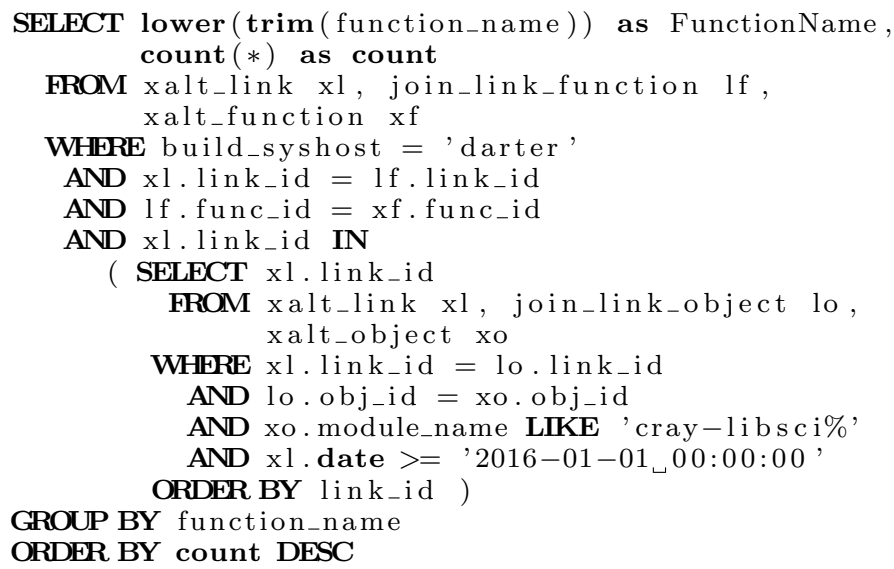

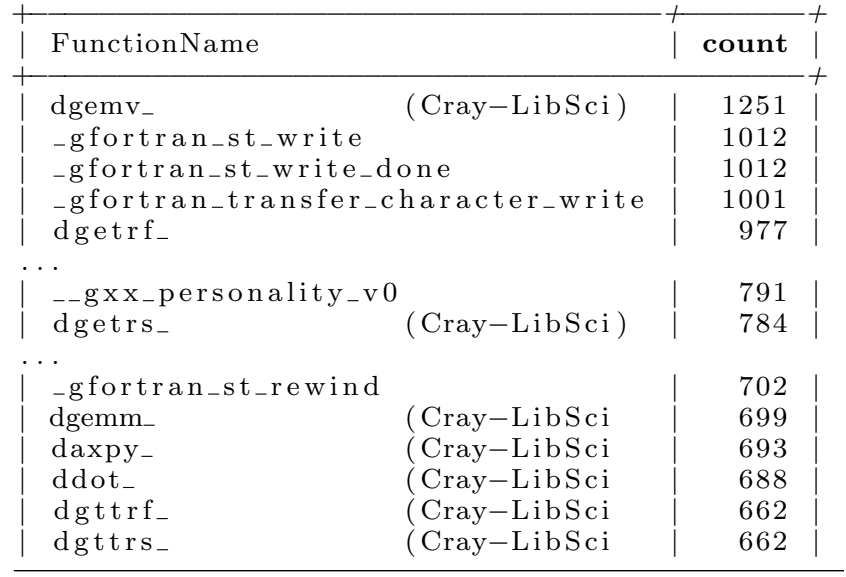

There are many potential benefits to having this kind of data and analysis. For example, by knowing which subroutines are more often used by applications and end-users, developers of numerical libraries can prioritize the subroutines they need to optimize. This information makes software development more efficient and impactful, especially in the world of HPC where hardware architecture is constantly changing and people resources are limited. Different numerical libraries also perform with different efficiency on different platform (for example, see [5]). Knowing which numerical libraries used by end-users for particular subroutines allows center staffs to assist end-users getting the most optimized subroutines for their applications, and advise them if they use an inappropriate library for certain subroutines.

\section{WEB INTERFACE}

To aid XALT users accessing the XALT data more easily, we recently developed a web-based interface to the XALT database, also known as the XALT-portal. There are four major pages on the XALT-portal. We describe those in this section along with some screenshots.

The first page is known as the Dashboard, giving a highlevel overview of users' activity as recorded by XALT. A screenshot of the XALT-portal Dashboard is shown on Figure 1. The data data displayed includes the top ten executa- bles by walltime, the number of "active users" (users who are either building or running executables), and the number of jobs submitted as recorded by XALT ${ }^{5}$. Figure 1 is a screenshot of the XALT-portal for NICS' system.

The rest of the pages allows one to get a detailed look of the XALT data from multiple interrogation entry points. For example, in Usage view, there are "Modules Usage" (a proxy for software and library usage, see [2]). Selecting one of the module name shows the versions available for that module (i.e. library), followed by the list of users who have used the selected library. One can then select any of the users to view the name of executables that the selected user have built linked to the selected library, and whether any of the executables has been run on the system. For a particular executable that has been run, one can select a particular run to see the environmental variables during the job submission for that executable. The list of subroutines called by that executable, and object files linked to the executable are also displayed. Figure 2 shows a screenshot with those information.

Another entry point to look at XALT data is by searching on a particular object path or executable name. XALTportal queries the database and then give the list of users who have either linked against that particular library, or built and run a particular executable name. This feature allows center's staff to easily obtain information in cases where they need to contact the users of a particular software.

Reproducible computational research requires that a complete, permanent record of the conditions and software environments used to compile and run applications to be available. This record therefore also contains the provenance, i.e. the history and origin, of the application in question. XALT already enhances reproducible research by creating this record, allowing users to know the provenance of their applications. In this paper [2], the authors outline how XALT can be used to provide software provenance. XALTportal implements the steps describes in [2], allowing any user to obtain the information on how a particular executable they run was built, including the object files linked to (libraries and their versions), and the subroutines called by the application. The conditions in which that executable was run are also available, including the list of all environmental variables and shared libraries loaded by the application (for a dynamically linked application). A commandline counterpart for this feature of XALT-portal is planned to make this feature even more accessible to user.

\section{CONCLUSION}

XALT is a tool that silently monitors activities in supercomputers to give accurate, detail, job-level information regarding software and application usage. XALT has been deployed on several HPC centers including the Texas Advanced Computing Center (TACC), the National Institute for Computational Sciences (NICS), and the National Center for Supercomputing Applications (NCSA). It is also being deployed on other supercomputers in XSEDE. Although the work that XALT does is transparent to the users and

\footnotetext{
${ }^{5}$ We note a caveat here that this information may be different from the record of a job accounting system (e.g. PBS, SLURM). As of this writing, XALT only records MPI jobs and therefore would miss serial and threaded (non-MPI) applications. Later version of XALT is planned to support non-MPI jobs.
} 
Active Users

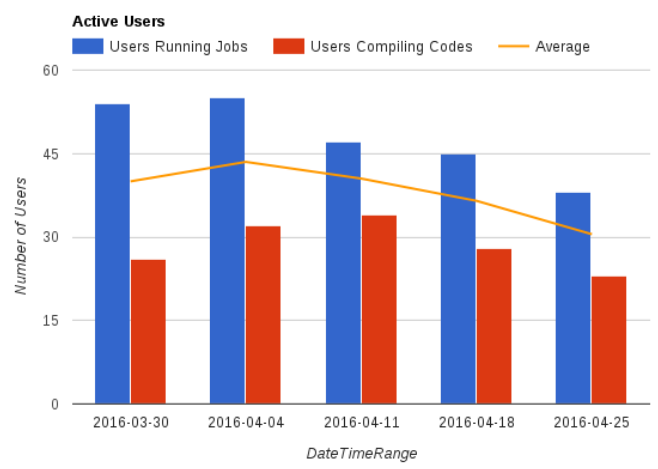

Top Ten Users using given resource (syshost)

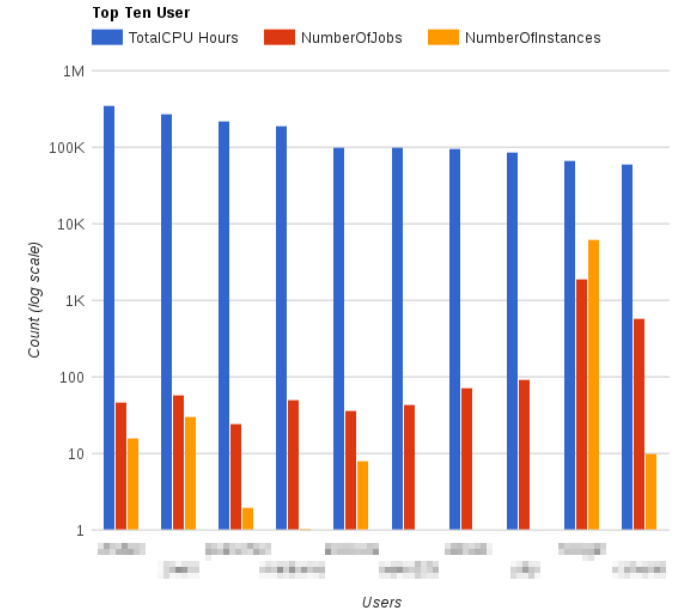

Jobs Submitted
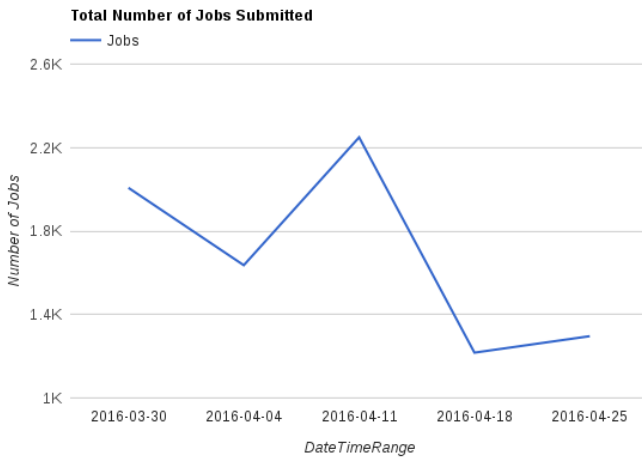

Top Ten Executables

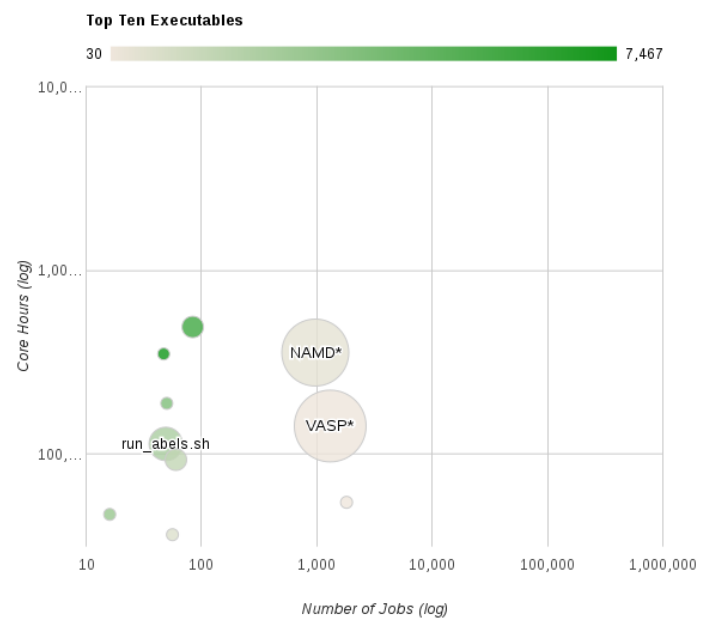

Compiler trend over given daterange.

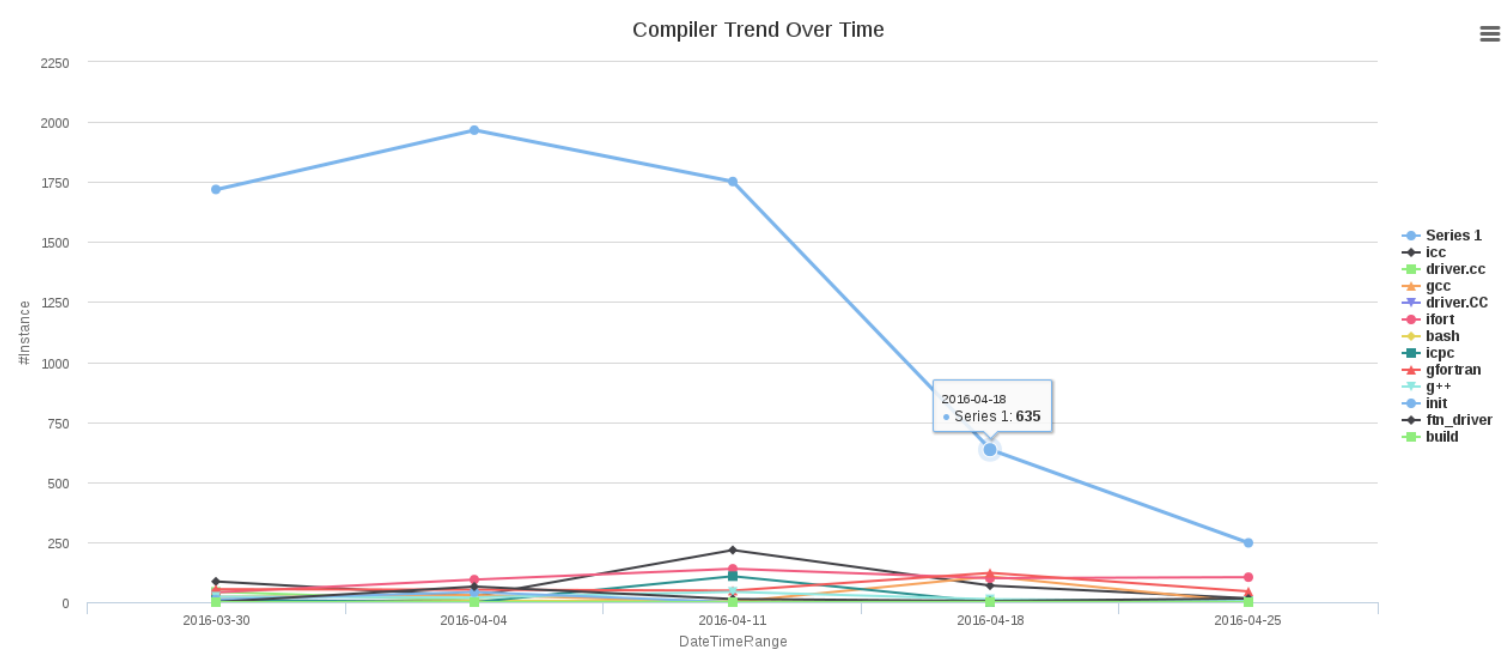

Figure 1: A screenshot of XALT-portal Dashboard view showing Active Users, Job Submitted, Top Ten Executables by Walltime, and compiler usage trend for the selected period. 


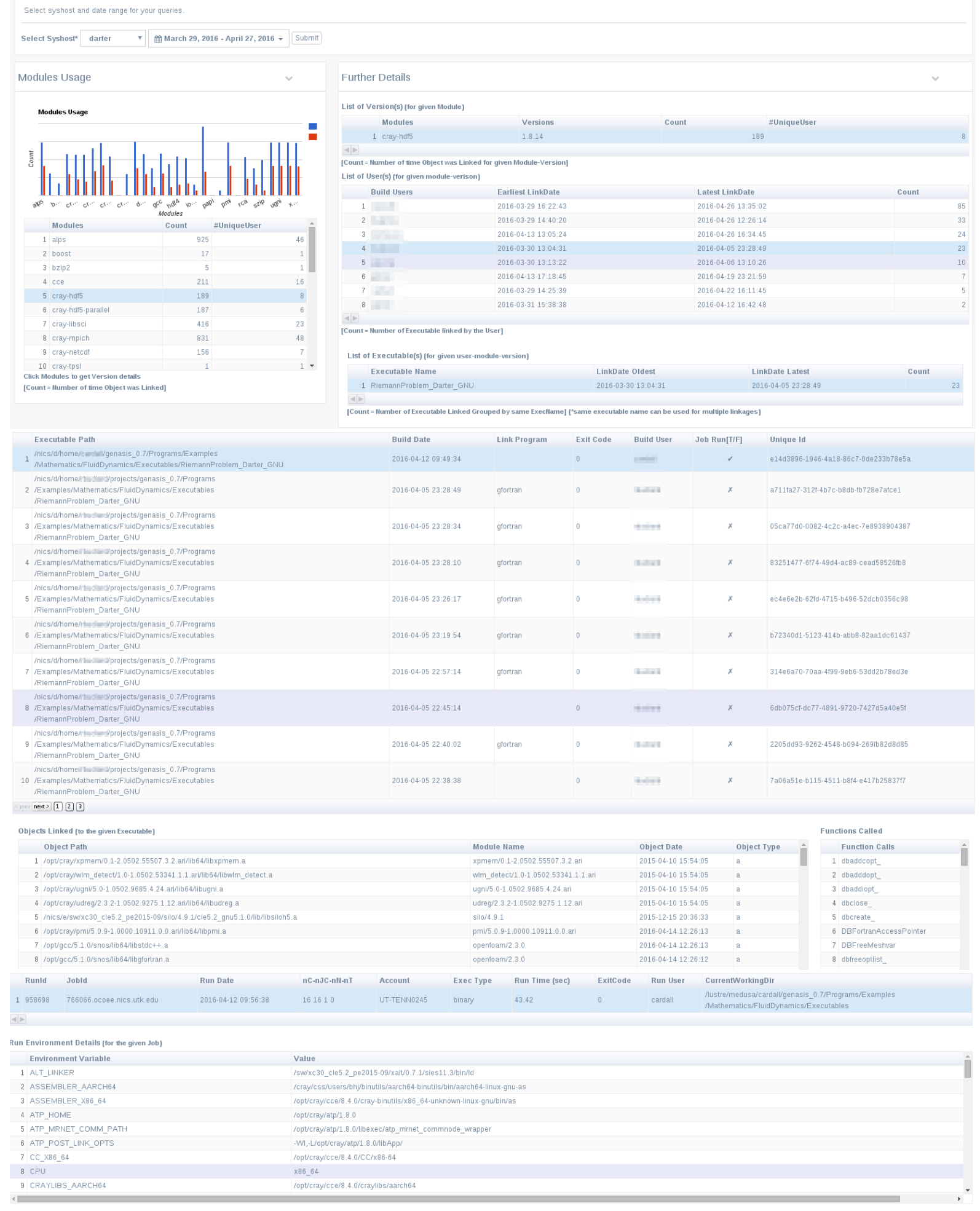

Figure 2: Screenshot of XALT-portal Usage view showing the list of modules (i.e. libraries) used in the selected time period for the selected system (Darter). Identifying information such as username has been obfuscated to protect users' privacy. On the top right panel we see the list of users who have used the cray-hdf5 library. Selecting a particular user gives a list of executables built by the user that were linked against the library, in this example: RiemannProblem_Darter_GNU. Multiple executables with the same name has been built by the user, but only one of them was ever run. This is a typical indicator of someone who is in a software development period. A list of object files linked by the executable, and subroutines called by the executable are also shown. Selecting the run executable shows the environment variables during the particular job submission (bottom panel). 
system administrators, its many benefits has increased productivity for all of the stakeholders an HPC system [6]. In this paper we have described a recent improvement in XALT the function tracking feature. This feature extends XALT capability to provide finer grain information and expands its benefit to users, system administrators, software developers, and vendors. We have also described our recent work in developing XALT-portal to give HPC staffs and users a more easily accessible interface to the XALT data with feature such as software provenance for reproducible research.

\section{Acknowledgment}

We would like to generally thank the XALT community in order to acknowledge the great feedback and bug reports.

This work was supported by the NSF award 1339690 entitled "Collaborative Research: SI2-SSE: XALT: Understanding the Software Needs of High End Computer Users." Argonne National Laboratory's work was supported by the U.S. Department of Energy, Office of Science, Advanced Scientific Computing Research, under contract DE-AC02$06 \mathrm{CH} 11357$.

This material is based upon work performed using computational resources provided by the University of Tennessee's Joint Institute for Computational Sciences and the Texas Advanced Computing Center (TACC) at the University of Texas at Austin.

\section{REFERENCES}

[1] K. Agrawal, M. Fahey, R. McLay, and D. James, "User environment tracking and problem dectection with XALT," in Proceedings of the First Workshop on HPC Tools for User Support (HUST14) Workshop held in Conjunction with the International Conference for High Performance Computing, Networking, Storage and Analysis (SC14), ser. HUST14, New Orleans, LA, November 2014.

[2] R. Budiardja, M. Fahey, R. McLay, P. M. Don, B. Hadri, and D. James, "Community Use of XALT in Its First Year in Production," in Proceedings of the Second International Workshop on HPC User Support Tools, ser. HUST '15. New York, NY, USA: ACM, 2015 , pp. $4: 1-4: 10$, dx.doi.org/10.1145/2834996.2835000. [Online]. Available: http://doi.acm.org/10.1145/2834996.2835000

[3] J. L. Furlani and P. W. Osel, "The Environment Modules Project," (retrived 2016), http://modules.sourceforge.net/.

[4] R. T. McLay, "Lmod Home Page," (updated 2016), tacc.utexas.edu/research-development/tacc-projects/ lmod.

[5] B. Hadri, H. You, and S. Moore, "Achieve better performance with peak on xsede resources," in Proceedings of the 1st Conference of the Extreme Science and Engineering Discovery Environment: Bridging from the eXtreme to the Campus and Beyond, ser. XSEDE '12. New York, NY, USA: ACM, 2012, pp. 10:1-10:8. [Online]. Available: http://doi.acm.org/10.1145/2335755.2335801

[6] D. James, R. McLay, S. Liu, R. T. Evans, W. L. Barth, A. Lamas-Linares, R. Budiardja, and M. Fahey, "Tales from the Trenches: Can User Support Tools Make a
Difference?" in Proceedings of the Second International Workshop on HPC User Support Tools, ser. HUST '15. New York, NY, USA: ACM, 2015, pp. 2:1--2:11. [Online]. Available:

http://doi.acm.org/10.1145/2834996.2834998 